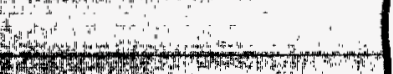

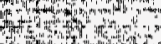

$\angle B L--38632$

$$
\text { CONF- } 960748--2
$$

ERNEST DRLANDI LAWRENCE BERKELEY NATIONAL LABDRATIRY

\title{
Local Vibrational Modes of Se-H Complexes in AlSb
}

M.D. McCluskey, L. Hsu, E.E. Haller,

W. Walukiewicz, and P. Becla

\section{Materials Sciences Division}

RECEIVED

September 1996

Presented at the

Seventh International

DEC 101996

OSTI

Conference on Shallow-

Level Centers in

Semiconductors,

Amsterdam, The Netherlands,

July 17-19, 1996, and to be published in the Proceedings

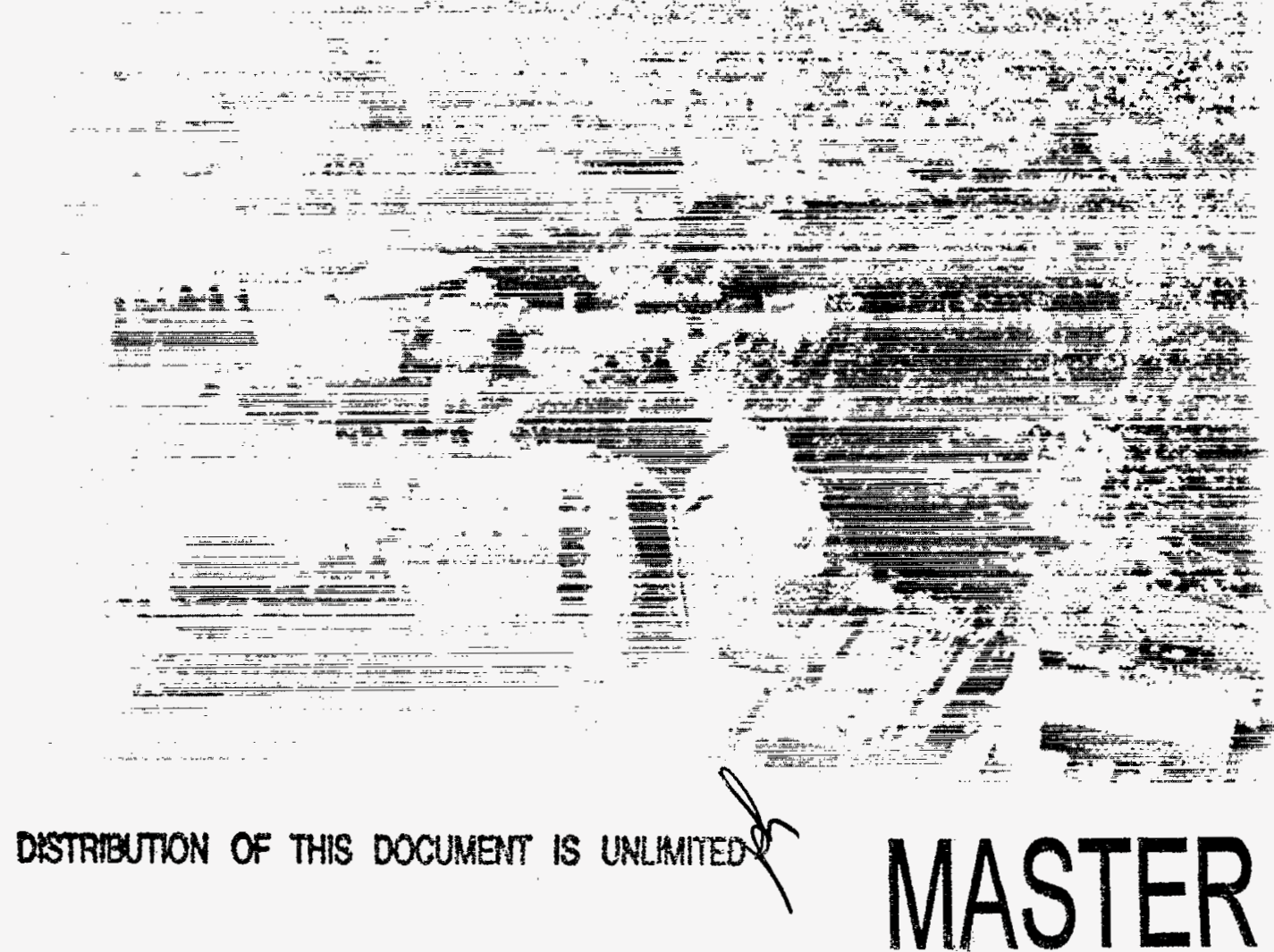




\section{DISCLAIMER}

This document was prepared as an account of work sponsored by the United States Government. While this document is believed to contain correct information, neither the United States Government nor any agency thereof, nor The Regents of the University of California, nor any of their employees, makes any warranty, express or implied, or assumes any legal responsibility for the accuracy, completeness, or usefulness of any information, apparatus, product, or process disclosed, or represents that its use would not infringe privately owned rights. Reference herein to any specific commercial product, process, or service by its trade name, trademark, manufacturer, or otherwise, does not necessarily constitute or imply its endorsement, recommendation, or favoring by the United States Government or any agency thereof, or The Regents of the University of California. The views and opinions of authors expressed herein do not necessarily state or reflect those of the United States Government or any agency thereof, or The Regents of the University of California.

Ernest Orlando Lawrence Berkeley National Laboratory is an equal opportunity employer. 


\section{DISCLAIMER}

Portions of this document may be illegible in electronic image products. Images are produced from the best available original document. 
LBL-38632

UC-404

\title{
Local Vibrational Modes of Se-H Complexes in AlSb
}

\author{
M.D. McCluskey, L. Hsu, and E.E. Haller, \\ Materials Sciences Division \\ Ernest Orlando Lawrence Berkeley National Laboratory \\ University of California \\ Berkeley, California 94720 \\ W. Walukiewicz \\ Center for Advanced Materials \\ Materials Sciences Division \\ Ernest Orlando Lawrence Berkeley National Laboratory \\ University of California \\ Berkeley, California 94720 \\ P. Becla \\ Department of Materials Science and Engineering \\ Massachusetts Institute of Technology \\ Cambridge, Massachusetts 02139
}

September 1996

This work was supported by the Director, Office of Energy Research, Office of Basic Energy Sciences, Materials Sciences Division, of the U.S. Department of Energy under Contract No. DE-AC03-76SF00098. 


\title{
LOCAL VIBRATIONAL MODES OF SE-H COMPLEXES IN AISb
}

\author{
M.D. MCCLUSKEY, L. HSU, E.E. HALLER \\ Lawrence Berkeley National Laboratory and University of California. \\ Berkeley, CA 94720 \\ W. WALUKIEWICZ \\ Center for Advanced Materials, Materials Science Division, Lawrence \\ Berkeley National Laboratory, Berkeley, CA 94720 \\ P. BECLA \\ Department of Materials Science and Engineering, Massachusetts Institute of \\ Tecinology, Cambridge, MA 02139
}

\begin{abstract}
Using infrared spectroscopy we have observed local vibrational modes (LVMs) arising from SeH complexes in AISb. At liquid-helium temperatures, hydrogenated AISb:Se samples have three stretch mode peaks at $1606.3,1608.6$, and $1615.7 \mathrm{~cm}^{-1}$, whereas deuteraled samples have only one peak at $1173.4 \mathrm{~cm}^{-1}$. The anomalous splitting of the Se-H stretch mode may be explained by a resonance between the stretch mode and two multi-phonon modes. As the temperature or pressure is increased, the stretch mode and multi-phonon modes show anti-crossing behavior.
\end{abstract}

\section{Introduction}

Hydrogen passivation of defects and impurities in semiconductors has been studied extensively over the past decade'. An important tool for studying the structure of hydrogen related complexes is local vibrational mode (LVM) spectroscopy, since hydrogen-related vibrational frequencies are typically much higher than the lattice phonon frequencies and the substitution of deuterium for hydrogen results in a significant isotope shift ${ }^{2}$. In AlSb, hydrogen diffuses rapidly through the bulk and passivates $\mathrm{Se}$ and $\mathrm{Te} \mathrm{DX}$ centers $^{3}$. The frequencies of the stretch and wag modes of $\mathrm{Se}-\mathrm{H}$ and $\mathrm{Te}-\mathrm{H}$ complexes provide evidence that the hydrogen attaches to a host aluminum atom in an antibonding [111] orientation. In this paper, we report on a resonance between the Se-H stretch mode in AlSb and an unknown mode of the lattice or the complex.

\section{Experimental Techniques}

The AlSb:Se samples were grown by the Czochrolski technique from selenium-doped melts. The growth was performed in a Sb-rich melt, with an atomic fraction $[\mathrm{Sb}] /([\mathrm{Sb}]+[\mathrm{Al}])=$ 0.515. Some of the samples were then placed in quartz ampoules with a $2 / 3$ atmosphere ambient of $\mathrm{H}_{2}$ or $\mathrm{D}_{2}$ and annealed at $900^{\circ} \mathrm{C}$ for $1 \mathrm{hr}$. After completion of the diffusion, the samples were quenched to room temperature by dropping the ampoules into ethylene glycol. 


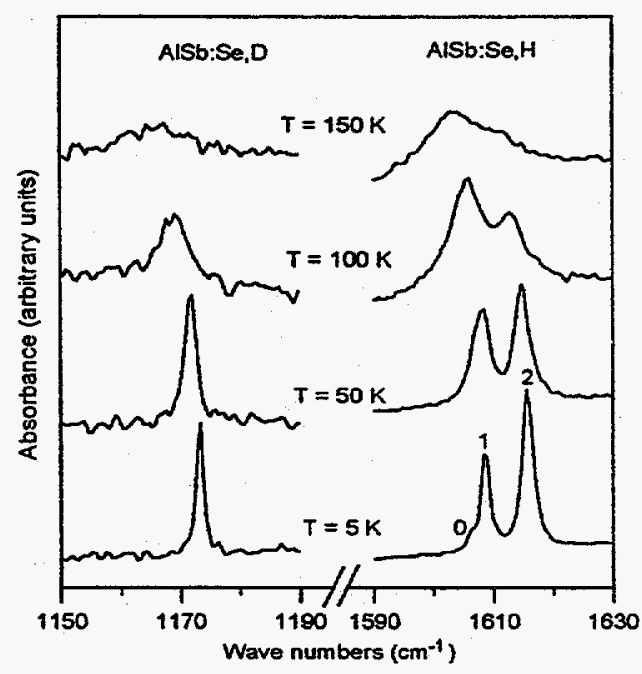

Figure 1: Temperature dependence of Se-D and Se-H stretch modes in AlSb.

Variable temperature infrared absorption spectra were obtained with a Bomem DA8 spectrometer with a $\mathrm{KBr}$ beamsplitter and an external mercury cadmium telluride (MCT) detector. The samples were placed in a Janis continuous-flow liquid helium cryostat with $\mathrm{ZnSe}$ windows. We used instrumental resolutions ranging from 0.1 to $1 \mathrm{~cm}^{-1}$ such that all the LVM peaks were fully resolved.

Variable pressure spectra were obtained with a Digilab 80-E spectrometer with a $\mathrm{KBr}$ beamsplitter and an instrumental resolution of $0.5 \mathrm{~cm}^{-1}$. To generate hydrostatic pressures up to $15 \mathrm{kbar}$, we used a modified Merrill-Basset diamond-anvil cell. The liquid immersion-technique was used to load the cell with liquid nitrogen. A light-concentrating cone focused the light through the diamonds and sample and into a $\mathrm{Ge}: \mathrm{Cu}$ photoconductor mounted directly behind the sample. We use the pressure dependence of the AlSb:C $C_{S b}$ LVM as a precise in situ calibration of the sample pressure. ${ }^{4}$.

\section{Results}

\section{I Temperature Dependence of LVMS}

As reported previously (Ref. 3), at liquid-helium temperatures, hydrogenated AlSb:Se has stretch mode peaks at 1608.6 and $1615.7 \mathrm{~cm}^{-1}$, whereas deuterated AlSb:Se has only one stretch mode at $1173.4 \mathrm{~cm}^{-1}$. In addition, there is a small Se-H peak at $1606.3 \mathrm{~cm}^{-1}$. The ratio of the three Se-H peak areas is constant from sample to sample, which suggests that they are not due to additional impurity complexes. In the following discussion, we provide 
evidence that the Se-H stretch mode interacts with two multi-phonon modes, giving rise to several absorption peaks. The Se-D stretch mode, which is far away from these modes, does not split. Although the two unknown modes may be overtones of other Se-H LVMs, for simplicity we refer to them as "multi-phonons."

The temperature dependence of the Se-H and Se-D stretch modes is shown in Fig. 1. The linewidth broadening and shift to lower frequency with increasing temperature is seen in numerous semiconductor systems and is caused by an anharmonic interaction between the localized mode and the extended lattice phonons $s^{5}$. Although the broadening obscures peak 0 , peaks 1 and 2 are clearly resolved up to a temperature of $100 \mathrm{~K}$. As the temperature increases, the area of peak 1 increases while the area of peak 2 decreases. The sum of the areas remains constant to within experimental error.

To explain these observations, we propose a model in which the Se-H stretch mode and a multi-phonon mode are nearly degenerate and interact with an energy $A$. The Hamiltonian is given by:

$$
H=\left[\begin{array}{cc}
\omega_{L M M} & A \\
A & \omega_{\text {phonon }}
\end{array}\right]
$$

The eigenvalues of this Hamiltonian are

$$
\omega_{ \pm}=\frac{1}{2}\left[\omega_{L V M}+\omega_{\text {phonon }} \pm \sqrt{\left(\omega_{L V M}-\omega_{\text {phonon }}\right)^{2}+4 A^{2}}\right]
$$

In our model, $A=3.45 \mathrm{~cm}^{-1}$. The minimum frequency difference between the two peaks is $2 A=6.9 \mathrm{~cm}^{-1}$. The eigenfunctions of the Hamiltonian (1) are linear combinations of an LVM and a multi-phonon,

$$
|\psi\rangle=a|L V M\rangle+b \mid \text { phonon }\rangle
$$

Since the multi-phonon mode is practically infrared inactive, the coefficient $a$ can be determined experimentally from the normalized area of each peak. For peak 1 , the lower frequency peak,

$$
|a|^{2}=A_{1} /\left(A_{1}+A_{2}\right)
$$

where $A_{1}$ and $A_{2}$ are the integrated areas of peaks 1 and 2 , respectively. The theoretical expression is given by:

$$
|a|^{2}=A^{2} / \sqrt{\left(\omega_{2 m}-\omega_{-}\right)^{2}+A^{2}}
$$


The temperature dependence of the unperturbed stretch mode is given by

$$
\omega_{L Y M}=1612.7-0.027 U(T) \text {, }
$$

where $U(T)$ is the mean vibrational energy of the lattice ${ }^{6}$ in cal/mole and $c_{2 m}$ is given in $\mathrm{cm}^{-1}$. The multi-phonon mode can be described by the empirical relation

$$
\omega_{\text {phonon }}=1611.5-16 /(\exp (380 / \mathrm{T})+1)
$$

As the temperature increases, the area of peak 1 increases as it becomes more "LVMlike" (Fig. 2). Conversely, the area of peak 2 decreases as it becomes more "phonon-like."
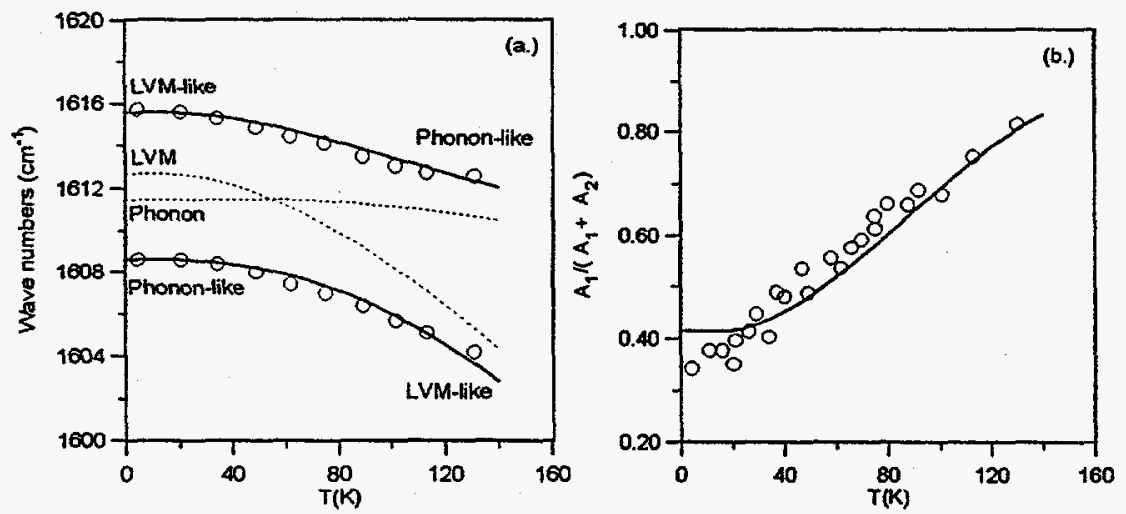

Figure 2: (a.) Se-H stretch modes as a function of temperature. The dashed lines are the unperturbed LVM and multi-phonon modes and the solid lines are the perturbed modes (Eq. 2). (b.) Normalized area of Se-H peak 1 (lower-frequency peak). The solid line is a plot of Eq. 5 .

\subsection{Pressure Dependence of LVMS}

An anti-crossing can also be observed when the hydrostatic pressure is increased. Varying the pressure has the advantage of not broadening the lines, so that both multi-phonon modes are resolved. As shown in Fig. 3, at pressures around $2 \mathrm{kbar}$, there are three absorption peaks. In our three-level system, the Hamiltonian is given by

$$
H=\left[\begin{array}{ccc}
\omega_{\text {LMM }} & A & B \\
A & \omega_{\text {phomonl }} & 0 \\
B & 0 & \omega_{\text {phomon. }}
\end{array}\right]
$$


where $A=3.45 \mathrm{~cm}^{-1}$ and $\mathrm{B}=1 \mathrm{~cm}^{-1}$. For simplicity we have neglected the interaction between the multi-phonon modes. The pressure dependence of the modes are given by

$$
\begin{aligned}
& \omega_{L I M}=1612.7+0.075 P \\
& \omega_{\text {phonon, } I}=1610.5+2.1 P \\
& \omega_{\text {phonon, } 2}=1605.8+2.1 P
\end{aligned}
$$

where $P$ is the pressure in kbar and the frequencies are in units of $\mathrm{cm}^{-1}$. The eigenvalues of the Hamiltonian (Eq. 8) are calculated using MATLAB. We obtain very good agreement between the model and experiment (Fig. 4).

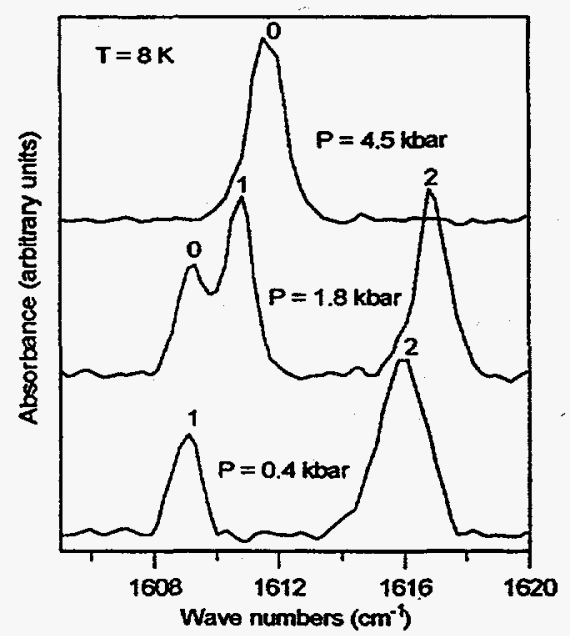

Figure 4: Se-H stretch mode peaks as a function of pressure.

\section{Conclusions}

Using variable temperature and pressure infrared spectroscopy, we have observed a resonance between the AlSb:Se,H stretch mode and two unknown modes. The unknown modes may be five optical phonons, since $5 \mathrm{X} \omega_{\mathrm{T}}(\Gamma) \sim 1610 \mathrm{~cm}^{-1}$ is very close to the observed frequencies. A second possibility is that the stretch mode interacts with the $\Gamma_{1}$ component of the wag mode fifth harmonic, but such a Fermi resonance would account for only one of the unknown modes. Clearly, this is an interesting problem which merits further investigation. 


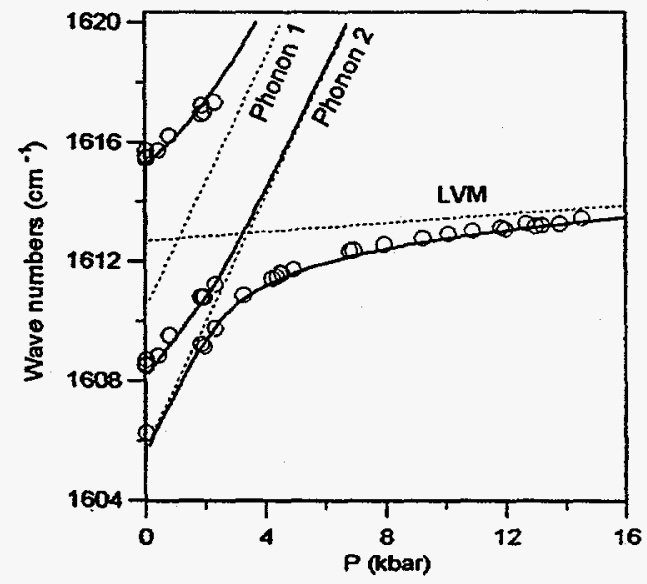

Figure 4: Se-H stretch mode peaks as a function of pressure. The dashed lines are the unperturbed LVM and multi-phonon modes and the solid lines are plots of the three-level theory (Eq. 8-9).

\section{Acknowledgments}

This work was supported by the Director, Office of Energy Research, Office of Basic Energy Sciences, Materials Science Division of the U.S. Department of Energy under Contract No. DE-AC03-76SF00098.

\section{References}

1. E.E. Haller, in Handbook on Seniconductors, edited by S. Mahajan (NorthHolland, Amsterdam, 1994), Vol. 3b, Ch. 20, p. 1515.

2. M. Stavola and S. J. Pearton, in Semiconductors and Senimetals, edited by J. I. Pankove and N. M. Johnson (Academic Press, Orlando, FL, 1991), Vol. 34, Ch. 8.

3. M.D. McCluskey, E.E. Haller, W. Walukiewicz, and P. Becla, Pliys Rev. B 53, 16297 (1996).

4. M.D. McCluskey, L. Hsu, L. Wang, E.E. Haller, submitted to Phys. Rev. B.

5. R.J. Elliot, W. Hayes, G.D. Jones, H.F. MacDonald, and C.T. Sennet, Proc. R. Soc. Lond. A289 1 (1965).

6. M.D. McCluskey, E.E. Haller, J. Walker, and N.M. Johnson, Phys. Rev. B 52, 11859 (1995). 